Itinerarios culturales y rutas turístico-culturales 05

\title{
Ejes del turismo cultural en la Eurorregión Galicia-Norte de Portugal
}

Xulio Pardellas. Profesor titular del Dpto. de Economía Aplicada. Universidade de Vigo

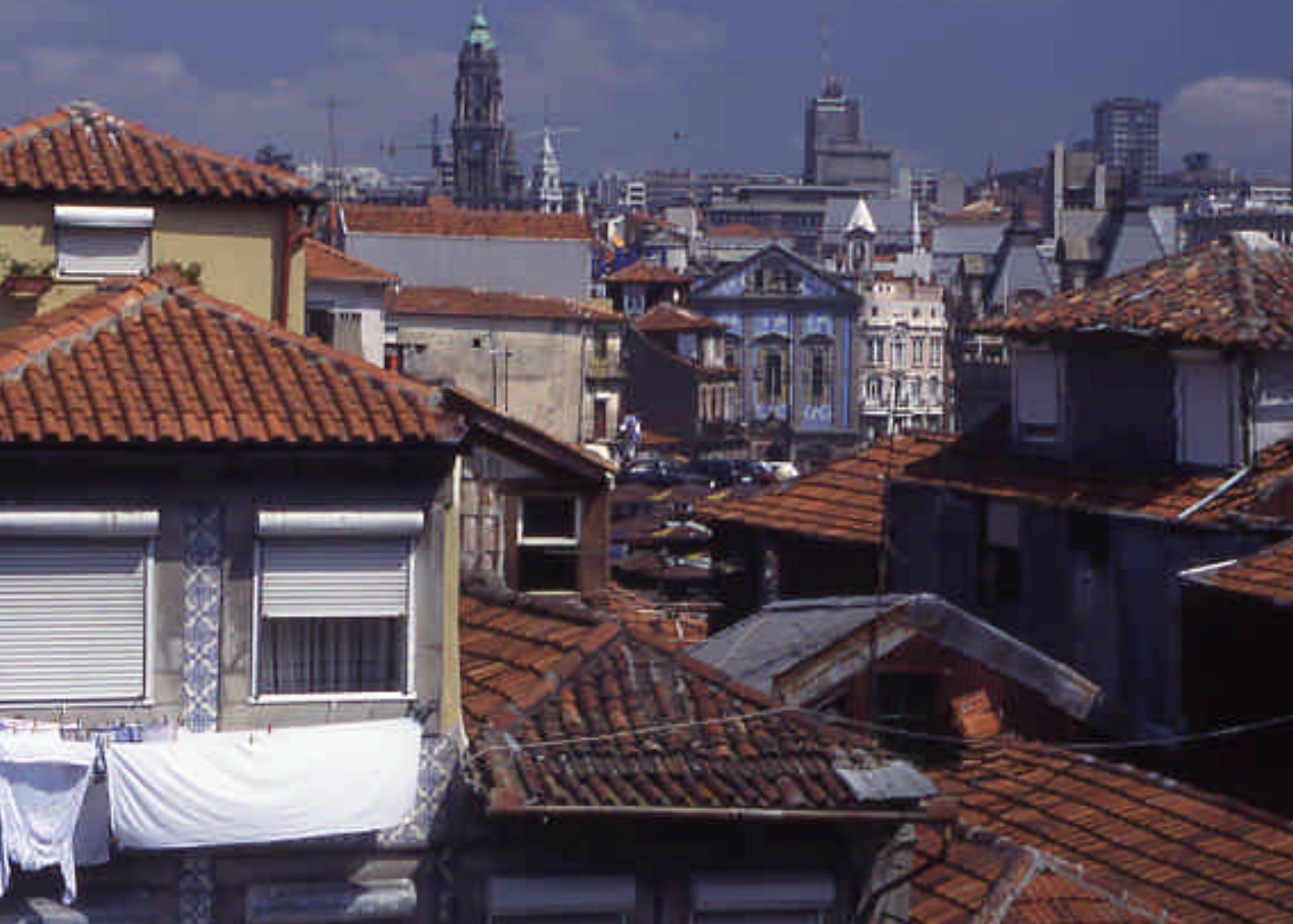




\section{Resumen}

Este trabajo resume los análisis y propuestas de varios estudios realizados por el autor en colaboración con investigadores de las Universidades do Minho y de Tras-OsMontes e Alto Douro, desde 1999. El objetivo principal de la investigación era conocer la situación de la actividad turística en el conjunto de la Eurorregión Galicia-Norte de Portugal y presentar propuestas de mejora desde el análisis de la totalidad del territorio. El artículo muestra brevemente el debate científico sobre los modelos aplicados a la planificación de destinos turísticos y, tras valorar los instrumentos jurídicos que podrían utilizarse desde las instituciones de la Eurorregión, analiza varios proyectos concretos referidos a la creación de itinerarios culturales susceptibles de ampliarse al conjunto de la Eurorregión, comentando sus efectos en el desarrollo local.

\section{Palabras clave}

Desarrollo local I Galicia I Itinerarios culturales I Norte de Portugal I Planificación I Turismo cultural

Q Vista general de Oporto (Portugal), Ciudad Patrimonio Mundial / Isabel Luque Ceballos
Nadie pone en duda la singular importancia económica del sector turístico en España, que en el 2004 se acercó al 11\% del PIB (AECIT, 2005 ), aportando un $10 \%$ del empleo, por lo que es habitual referirse al turismo como la primera industria del país (ANTÓN y MONFORT, 2002). En Portugal los datos son menos esclarecedores; sin embargo, podemos afirmar, con los datos del INE (2004), que su aportación al PIB puede acercarse fácilmente al $7 \%$, aunque en los dos casos esta actividad no of rece una distribución geográfica regular, existiendo una fuerte concentración en ciertas áreas como el litoral mediterráneo 0 las Islas Baleares y Canarias, en España, y el Algarve, en Portugal.

Los problemas estadísticos son mayores cuando nos acercamos a una desagregación regional, relativamente cubierta en el caso de Galicia, pero con notables dificultades en la Regiâo Norte. En todo caso, la definición y planificación de los destinos turísticos es un tema de especial importancia para las administraciones regionales, donde a menudo aparece propuesta y gestionada desde otros enfoques relacionados con el turismo, pero no siempre de forma directa, tales como los usos del suelo, la gestión del medio natural o el equipamiento en infraestructur ras (MARTÍNEZ, 2000). La mayoría de estos enfoques tiene como objetivos principales otros temas y se ejecutan con independencia de las iniciativas de la planificación turística (DREDGE, 1999).

\section{LA PLANIFICACIÓN TURÍSTICA Y EL TERRITORIO}

Este trabajo resume los análisis y propuestas de varios estudios realizados por el autor en colaboración con investigadores de las Universidades do Minho y de Tras-Os-Montes e Alto Douro, desde 1999. El objetivo principal de la investigación era conocer la situación de la actividad turística en el conjunto de la Eurorregión y presentar propuestas de mejora, lo que en rigor implicaba proponer modelos de 


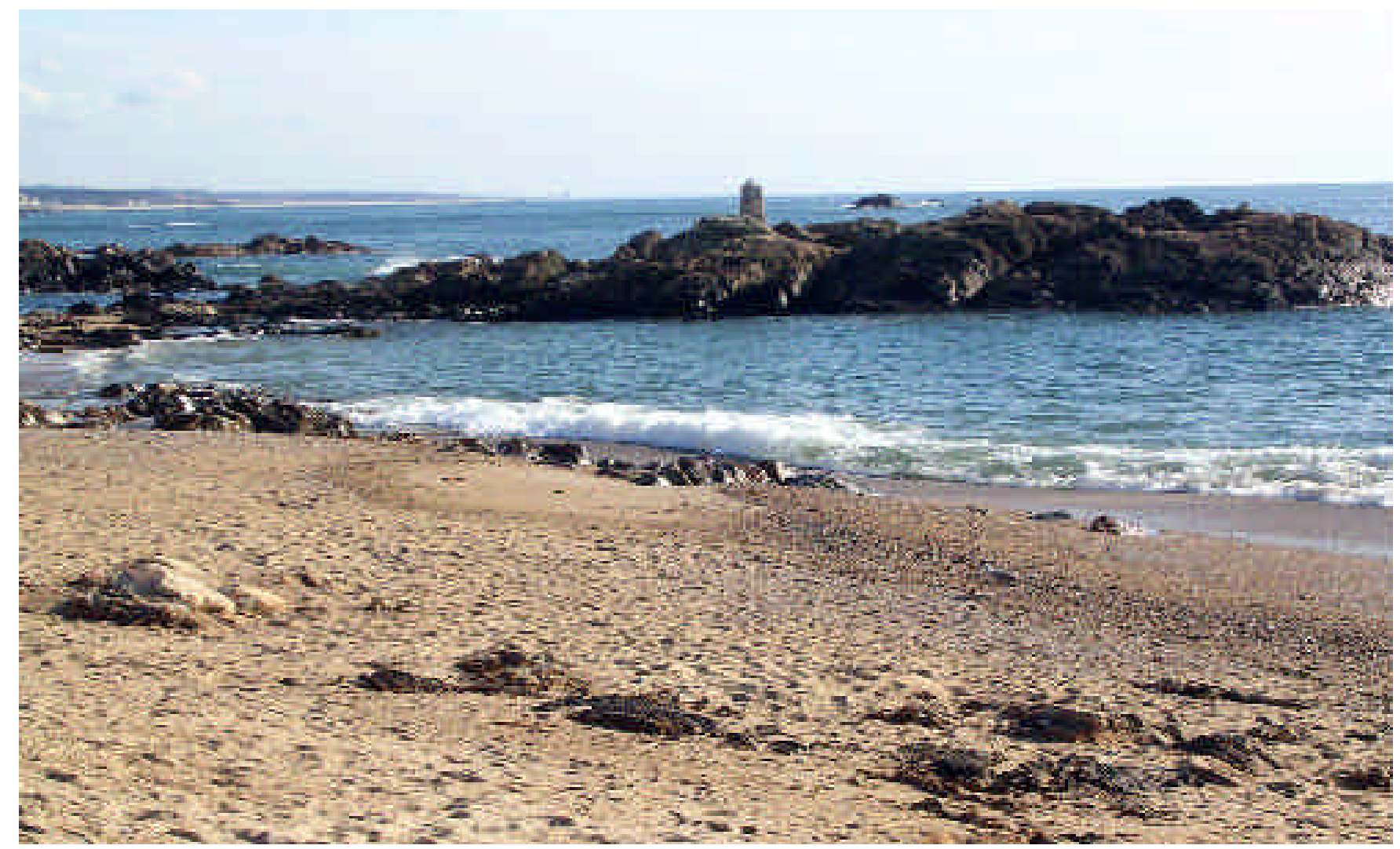

W Foz do Douro (área metropolitana de Oporto) / JAvier Soto

planificación. Se propuso un análisis conjunto de la totalidad del territorio utilizando aportaciones conceptuales que desde diversos enfoques permitían elaborar la hipótesis de un destino turístico común con mayor potencial de atracción de demanda que la agregación simple de las capacidades y recursos de Galicia y el Norte de Portugal.

Esta percepción de destino común en el comportamiento de los turistas aparecía con cierta claridad en estudios realizados en el área fronteriza del río Miño entre Galicia y el Norte de Portugal. Más de un $90 \%$ de los que se alojaron en hoteles de los municipios gallegos visitaron pueblos y ciudades de Portugal, y viceversa, en un 70\% (PARDELLAS, 2001 CADIMA, 2002). Sin embargo, no existen datos que permitan afirmar la continuidad de ese comportamiento en los municipios situados a más de $30 \mathrm{~km}$ al norte y sur de la frontera (DE MIGUEL, 2000).

La decisión metodológica de los investigadores tuvo en cuenta también la actividad de coordinación administrativa de la Comunidad de Trabajo Galicia-Norte de Portugal, creada en 1989 y encargada de avanzar en la nueva configuración del territorio de la Eurorregión, así como en la construcción de una autoridad administrativa conjunta (CAMPESINO, 1996; PARDELLAS, 2002), sobre la que reflexionaremos más adelante. Por otra parte, ese enfoque coincide plenamente con la recomendación de la propia Comisión Europea (1999), en el sentido de que las tendencias de desarrollo territorial deben estar basadas en la integración económica y la cooperación, y al mismo tiempo en la voluntad de las autoridades regionales y locales.

Desde esa perspectiva, el análisis territo rial y los problemas del desarro llo local deben interpretarse espacialmente, incluso considerando al territorio no como la base física de las actividades económicas, sino como un recurso endógeno más (FUÁ, 1988; GAROFOLI, 1992; VÁZQUEZ BARQUERO,1999). Es posible encontrar en la literatura científica un cierto número de referencias en las que se intenta vincular el turismo con la planificación local, sobre todo en áreas rurales (ASHWORTH and DIETVORST, 1995; FYALL and GARROD, 1998; SWARBROOKE, 1999; ROBERTS and HALL, 2001), y con especial énfasis en la sostenibilidad y en la necesidad de integrar el turismo en los sistemas productivos locales (MANNING, 1999; RYAN, 1999), lo que significa establecer una interrelación territorio-recursos, buscando externalidades positivas.

Aunque no es objetivo de este trabajo profundizar en el debate sobre el desarrollo endógeno, las citas son relevantes dado que, por un lado, los recursos turísticos son siempre endógenos, y por otro lado, es raro 
encontrar análisis críticos sobre el tipo de estrategias espaciales en los niveles más altos del gobierno, fuera de los planes de desarrollo regional presentados por los países europeos al FEDER (COFFEY and POLĖSE, 1985; AYDALOT, 1986; MAILLAT , 1998). En España, por ejemplo, este vacío ha dado lugar a serios problemas de interpretación y aplicación de proyectos de desarrollo en los que los planificadores no han considerado las características específicas del desarrollo turístico (VALDÉS, 1996). El problema crece si tenemos en cuenta que el territorio estudiado pertenece a dos naciones y que mientras una de ellas tiene un nivel administrativo regional (Galicia), la otra mantiene una única administración nacional (Portugal). Desde luego, hay interesantes análisis metodológicos de planificación turística (GETZ, 1988; INSKEEP 1991; VERA , 1997), pero sus principales críticas apuntan a la fragmentación espacial PEARCE, 1995), o a la excesiva relevancia de algunos conceptos geográficos como la interacción espacial entre componentes, la jerarquía nodal o los circuitos turísticos, por su limitada capacidad para identificar un modelo preferente del uso del suelo y de los recursos (FAGANCE, 1995).

Tradicionalmente, el campo de interés de los planificadores ha estado muy vinculado a los usos residenciales, comerciales e industriales, no siempre bien interrelacionados, ignorando con frecuencia que la actividad turística implica a un elevado número de sectores económicos y al mismo tiempo se interrelaciona con diferentes usos del suelo. Por otra parte, nuevos valores sociales que afectan a la conservación ambiental 0 al desarrollo sostenible están influyendo sobre las administraciones y especialmente sobre los planificadores (BUSBY and CURTIN, 1999; SWARBROOKE and HORNER, 1999), lo que tiene una notable relevancia en las propuestas de metodologías, conceptos, modelos y teorías para enf rentar con rigor los problemas de los destinos turísticos y dar las soluciones más adecuadas para facilitar el diseño de las regiones de destino. Esta necesidad es de singular importancia en el territorio estudiado, que requiere respuestas prácticas en la elaboración de un modelo de configuración espacial que simplifique los flujos de turistas hacia la Eurorregión como destino y dentro de ella, y al mismo tiempo, que ese modelo pueda ser aceptado por las dos administraciones que gestionan las dos regiones, para que exista coordinación entre sus actuaciones y los visitantes perciban el sentido de un destino turístico común.

Desde mediados de los 70 y con la aprobación del FEDER, la literatura sobre planificación ha crecido notablemente, existiendo un elevado número de estudios y análisis críticos tanto sobre los modelos de uso de los recursos, como de los propios instrumentos de planificación (CAMPBELL y FAINSTEIN, 1996). En la literatura turística podemos diferenciar los instrumentos que se ocupan de la naturaleza de los procesos de planificación, centrándose en la decisión y la política, con aportaciones muy relevantes de Getz (1986) y de Inskeep (1988 y 1991), que tienen una relativa importancia para este trabajo, pero sólo como

\section{La vecindad de lugares con características físicas semejantes debe tenerse muy en cuenta para identificar con precisión un destino}

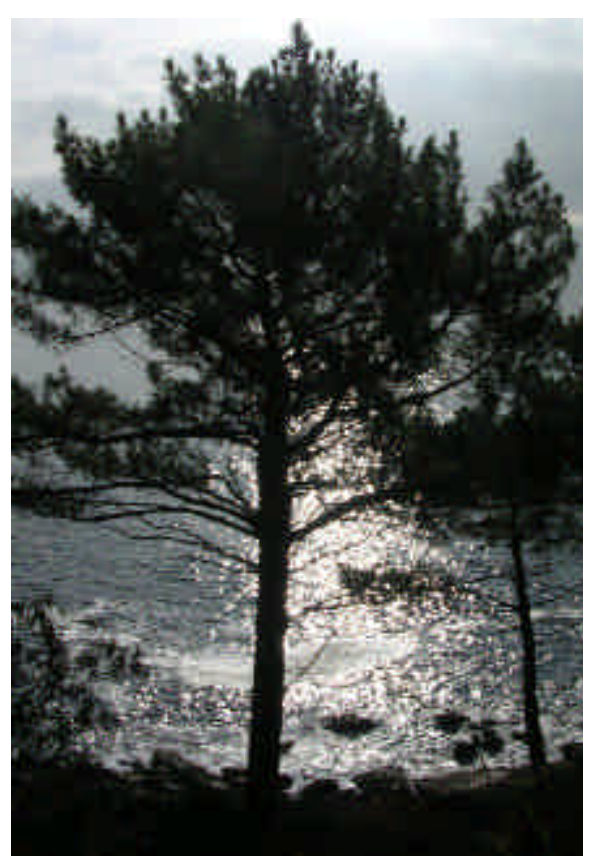

W Finisterre (Costa da Morte) / Esther Sanjuán 
referencia metodológica. Por otra parte, son más numerosos los estudios que proponen instrumentos funcionales, la mayoría desde un enfoque descriptivo, que en sus aportaciones más interesantes para este artículo intentan explicar la relación dinámica entre el comportamiento humano y las formas de asentamiento, como podemos ver en el modelo centro-periferia de Britton (1980), en el análisis de las pautas de comportamiento en viaje aportado por Lundgren (1982), o en el modelo de viaje multidestino de Lue, Crompton y Fesenmaier (1993), que propone cinco modelos de viaje: destino único, campo base, en ruta, tour regional y viaje encadenado.

Otro enfoque es el análisis de los instrumentos normativos buscando la conexión entre valores humanos y formas de asentamiento. Probablemente los estudios de mayor relevancia para este trabajo son los de Gunn $(1972,1993)$ alrededor del concepto de planificación regional, proponiendo una región destino con cinco elementos básicos: la definición precisa de los límites regionales, un acceso desde los mercados con corredores de circulación interna, unos complejos de atracción, un hinterland no atractivo y entradas a la región. Complementariamente, Pearce (1995) sintetiza varios trabajos de planificación y análisis desde la perspectiva de la evolución de los destinos, entre los que destacan el modelo de evolución espacio-temporal, que incorpora los cambios en el grado de participación local, y con mucho mayor interés para este estudio, los estudios de Opperman (1993) sobre el papel de los turistas informales para abrir una región destino a los mercados "formales", y de Leiper (1995) que identifica las características y condiciones para que un destino sea atractivo.

Es cierto que la mayoría de los análisis citados se refiere a casos concretos; por ejemplo, los modelos que se ocupan de las pautas de comportamiento o los viajes multidestino se han desarrollado principalmente sobre datos de Norteamérica y tienen una utilidad limitada para planificar regiones de Europa, donde los factores espaciales y la vecindad de destinos con características físicas semejantes deben tenerse muy en cuenta para identificar con precisión un des. tino. Desde otra visión, el análisis de los instrumentos funcionales y evolutivos se ha desarrollado con datos de estudios empíricos, tratando de explicar la comprensión de las características de un destino y aspectos de su desarrollo, o también los comportamientos de los turistas, lo que proporciona al planificador una importante información previa a su trabajo, pero le ayuda poco en la identificación de preferencias en función de objetivos territoriales.

Sin embargo, a pesar de esas limitaciones, podemos destacar ideas interesantes en esos modelos. Sin duda las de mayor relevancia aparecen en las aportaciones de Gunn (1972 y 1993) que contribuyen a la descripción de la estructura física de las regiones destino. Sus conclusiones son ampliamente citadas, siendo uno de los pocos que se ha centrado en la descripción y desarrollo de modelos estructurales de destinos turísticos. En su concepto más elaborado de planificación regional, Gunn (1972 y 1993) identifica los cinco conceptos clave antes citados: frontera, acceso y corredor interno, complejos de atracción, regiones vecinas no atractivas y entradas. Desde otros enfoques, y casi simultáneamente, los citados Lue, Crompton y Fesenmaier (1993) identifican cinco modelos de viajes, destacando el modelo de viaje encadenado hacia "focos" de atracción y los estudios más recientes de Swarbrooke y Horner (1999) y de Pizam y Mansfeld (2000) contrastan el modelo de comportamiento de los turistas con respecto a destinos donde existan o puedan crearse nodos de atracciones y estructuras de recorrido circular. Coincidiendo con ese análisis, Leiper (1995) identifica tres componentes de un sistema de atracción: un núcleo, un turista y un marcador. El núcleo es el elemento central de las atracciones turísticas, el turista es quien viaja y mantiene un contacto personal con los lugares visitados y el marcador es un instrumento de información para el turista.

En este contexto, el trabajo de Dredge (1999) aporta una interesante base teórica, que sintetiza y reestructura los anteriores, en especial las ideas de Gunn y Leiper, con una propuesta en la que destacan tres supuestos fundamentales como base de su modelo: que los mercados emisores de turismo y las regiones destino sean entidades geográficas separadas, que la naturaleza compleja y de distintos niveles de los destinos requiera una estructura jerárquica pero flexible, y que el modelo de planificación y diseño comprenda una región de destino, mercados emisores de turismo, nodos, distritos, rutas circulatorias y puertas (1999:403).

Desde las aportaciones metodológicas citadas, pensamos que es posible elaborar un modelo de análisis de la Eurorregión GaliciaNorte de Portugal como destino turístico conjunto, ya en el medio plazo, proponiendo, entre otras posibilidades, la de modelos de itinerarios axiales y circulares que pongan en valor los recursos de ambas regiones.

\section{LA COMUNIDAD DE TRABAJO GALICIA-NORTE DE PORTUGAL Y EL SISTEMA URBANO DE LA EURORREGIÓN}

A penas remontándonos al final de la última guerra mundial, la primera institución europeísta que apostó por la cooperación transfronteriza e interregional fue el Consejo de Europa, fundado en Londres en 1949, que asentó el concepto de región como parte integrante del proceso de construcción europea y que dio pasos claros en esa dirección con la iniciativa de crear en 1957 la Conferencia Europea de Poderes Locales, denominada, desde 1994, Congreso de Poderes Locales y Regionales de Europa (ROJO, 1996), fundando previamente, en 1971, la Asociación de Regiones 
Fronterizas Europeas, que más tarde daría lugar a la Asamblea de Regiones de Europa (ARE) en 1987, desempeñando un papel determinante para conseguir que, ya dentro del proceso de integración, las regiones tuviesen representación institucional en la UE mediante el Comité de la Regiones, sancionado en el Tratado de Maastricht, en 1992.

El impacto concreto de este proceso en el área territorial del estudio presenta, sin embargo, diferencias notables que afectan a la idoneidad del modelo jurídico necesario para garantizar la operatividad del diseño del destino turístico en la Eurorregión. Destacaremos en primer lugar las dif erentes estructuras administrativas existentes: mientras que España constituye actualmente un Estado dotado de una gran descentralización política, normativa y administrativa, por el contrario, Portugal es uno de los Estados más centralizados de la Unión Europea, tanto política como administrativamente. Este aspecto origina problemas para incrementar la cooperación transfronteriza y ahondar en una mayor integración eurorregional al aparecer una singular asimetría institucional en los interlocutores que deberían activar, incentivar y gestionar tal proceso.

Podemos ver así que Portugal crea en 1979 la Comissâo de Coordenaçâo da Regiâo Norte (CCRN, denominada a partir de 2003, de Coordinaçâo e Desenvolvimento, CCDRN), sin competencias legis lativas ni autogobierno, pero con una muy importante capacidad de acción por la gestión de fondos comunitarios y por su papel de control y asesoramiento técnico a los poderes locales en lo que respecta al desarrollo regional (DOMÍNGUEZ y VENADE, 2004). Siguiendo otro modelo, España sanciona en la Constitución de 1978 el Estado de las Autonomías, y por la Ley Orgánica de 1981 se promulga el Estatuto de Autonomía de Galicia, que en su artículo 27 establece la competencia exclusiva en la promoción y ordenación del turismo, lo que aparecerá expresado más tarde, en 1997, en la aprobación por el Parlamento Galego de la Ley de Ordenación y Promoción del Turismo en Galicia, modificada en algunos conceptos por la Ley $10 / 2004$, del mismo nombre.

Partiendo de esta importante diferencia competencial entre las dos regiones, el debate debe centrarse en el actual proceso de cooperación y en su capacidad para crear instrumentos válidos con el objetivo de coordinar acciones institucionales comunes. Campesino (1996), Cadima (1996) y Pardellas $(2002,2003)$ analizan desde la perspectiva geográ fica y económica el proceso de evolución y las competencias de la Comunidad de Trabajo Galicia-Norte de Portugal, creada en 1991 por la Xunta de Galicia y la Comissâo de Coordenaçâo da Regiâo Norte, como punto de partida para la constitución efectiva de la Eurorregión, y destacan algunos resultados positivos en la nueva configuración del territorio fronterizo, especialmente en lo que se refiere a la accesibilidad física entre las dos regiones.

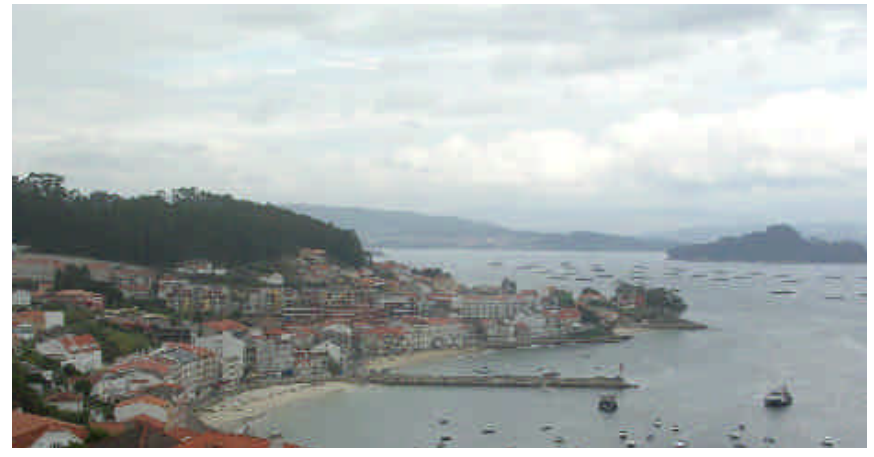

W Sanxenxo (Pontevedra), población situada en las Rías Baixas, contorno paisajístico que forma parte de una de las zonas que componen la Eurorregión denominada tierras del litoral / Esther SAnJuÁN 
X Viana do Castelo (norte de Portugal), costa atlántica entre Minho y Douro / Isabel Dugo

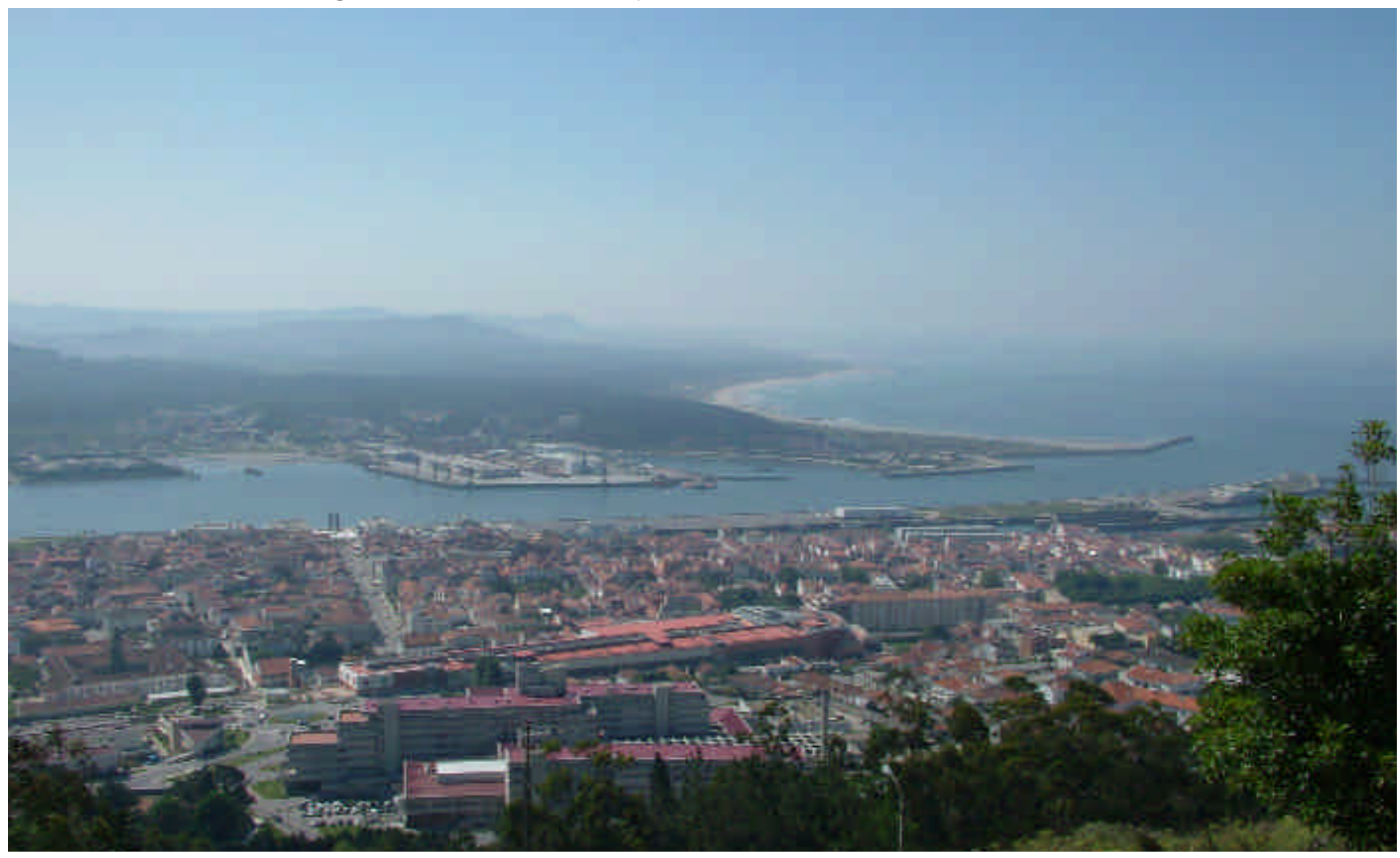

066 MONOGRÁFICOI PH Boletín del Instituto Andaluz del Patrimonio Histórico I no 60 I noviembre 2006 I pp. 60-73 
Desde otro enfoque, Venade (2004:62) plantea dudas jurídicas en la operatividad del "acuerdo" de creación de la Comunidad de Trabajo, subrayando que se trata de "un compromiso de carácter no normativo, o sea, no tiene una naturaleza jurídica definida y mucho menos vinculante desde el punto de vista jurídico-internacional". Para este autor, el acuerdo expresa un compromiso de valor meramente declarativo, e incluso la propia estructura orgánica de la Comisión de Trabajo no incluye una disposición de carácter doctrinario que aclare las funciones de sus órganos de dirección. Con todo, el mismo autor reconoce que la firma en Valencia en octubre de 2002 de la Convención entre el Reino de España y la República de Portugal sobre Cooperación Transfronteriza (que entró en vigor el 1 de enero de 2004 , siendo conocida ya como Convención de Valencia) abre un nuevo marco de cooperación a través de una "disciplina jurídica apropiada" (2004:70), que impone un protocolo específico a las instancias y entidades territoriales que estén interesadas en concretar aquella cooperación.

Pero en el momento actual y ya con esta perspectiva histórica, el proceso presenta indudables avances, y aunque el propio término de Eurorregión no corresponde a un concepto jurídico suficientemente preciso (VENADE, 2004), en la terminología del Consejo de Europa puede designar a cualquier acuerdo de cooperación transfronteriza con el objetivo de promocionar intereses comunes que beneficien a las poblaciones de ambas regiones, por lo que el territorio de la Comunidad de Trabajo puede considerarse en rigor una Eurorregión y varios estudios académicos así lo expresan (HERRERO, 2002). Por otra parte, la incorporación a la Comunidad de Trabajo en 2001 del Eixo Atlántico, integrado por las 18 ciudades más importantes de ambas regiones, ha completado el abanico de instituciones que pueden aportar una colaboración decisiva al funcionamiento ef ectivo de la Eurorregión.

Situados de nuevo en el territorio objeto del estudio, es preciso destacar que una de las diez comisiones sectoriales de la Comunidad de Trabajo se denomina "Patrimonio y Turismo" $y$, aun admitiendo que el turismo no es todavía una prioridad en el trabajo de aquella comunidad territorial, sin embargo, la Comunidad de Trabajo tiene competencias para solicitar y usar fondos comunitarios, por lo que tanto de facto, como de iure, configura un instrumento institucional jurídicamente válido para protagonizar acuerdos concretos de coordinación en el diseño y operatividad de un destino turístico transfronterizo como el aquí presentado.

Por su parte, la estructura territorial y urbana no es ajena a la evolución de las instituciones y desde la integración de España y Portugal en el proceso de construcción europea, en 1985, tanto la orientación de las infraestructuras construidas con el apoyo financiero de la UE (en especial desde los programas INTERREG), como las interrelaciones que se han ido construyendo entre los agentes económicos y sociales, están produciendo una clara reconfiguración del territorio $y$, desde luego, están promoviendo nuevos cauces de cooperación institucional con efectos en el sistema urbano, en el desarrollo local y en el uso de los diferentes recursos para favorecer la actividad turística.

Desde la perspectiva puramente geográfica, el territorio de la Eurorregión lo podemos dividir esquemáticamente en 2 grandes zonas, que a su vez se subdividen en contornos paisajísticos diferenciados:

j Las tierras del litoral, con amplia urbanización, elevada densidad y movilidad espontánea de la población:

\section{1.-Litoral Norte (As Mariñas)}

2.-Litoral del Golfo Artabro (A Coruña-Ferrol)

\section{3.-La Costa da Morte}

4.-Las Rías Baixas (Vilagarcía, Pontevedra, Vigo)

5.-El Baixo Miño gallego-Vale do Minho portugués

6.-La costa atlántica entre Minho y Douro (Viana do Castelo)

7.-Los valles de Cávado y Ave (Braga-Guimarães)

8.-La foz del Douro (Area Metropolitana de Porto)

j Las tierras del interior, con una pérdida demográfica importante en las últimas décadas, un proceso de envejecimiento y una pérdida de productividad económica:

\section{1.-Las sierras septentrionales de Galicia}

2.-La Depresión meridiana de Galicia y las montañas occidentales (Santiago)

3.-La Terra Chá y el valle del Miño (Lugo, Monforte)

4.-Las cuencas y depresiones del interior (Ourense, A Lima, Chaves-Verín)

5.-Las sierras orientales de Galicia

6.-Tras-Os-Montes (Bragança)

7.-El Vale do Douro (Peso da Régua, Vila Real)

Sobre este extenso y diverso territorio se localizan reservas ecológicas de interés nacional (Illas atlánticas, Parque do Gêrés) y regional (Dunas de Corrubedo, Parque do monte Aloia), monumentos históricos de notable interés (las fortalezas abaluartadas y enfrentadas en el Miño, TuiValença, Goián-Vilanova de Cerveira), ciudades patrimonio de la humanidad (Santiago, Lugo, Porto), y sobre todo un sistema de ciudades y de relaciones que configuran con claridad dos ejes bien diferenciados, coincidentes con las dos áreas antes citadas.

Existe un potente eje litoral, donde se encuentran las principales ciudades con fuertes interrelaciones, y en paralelo, otro eje interior, con relaciones más débiles, pero con un singular potencial económico, especialmente en el ámbito turístico (PARDELLAS, 2002 ), que comentaremos en el siguiente apartado. 


\section{EL MODELO DE RUTAS E ITINERARIOS CULTURALES}

En los tiempos modernos, el modelo turístico de itinerario cultural, entendido como un viaje relativamente corto en el tiempo y en el espacio con objetivos múltiples de tiempo libre y curiosidad, aparece a finales del s. XVIII con la denominación de Grand Tour (EZQUERRA, 2001) como un viaje educativo y cultural para jóvenes ingleses y franceses de clase alta que también llegó a conocerse como "Voyage en Italie", puesto que el destino casi exclusivo de aquellos jóvenes era justamente la península italiana y sus ciudades. En aquel tiempo, España no fue incluida en Grand Tour, mientras que los Países Bajos, el sur de Francia y Austria fueron integrándose en el interés de esa nueva clase de turistas. Tendremos que esperar a mediados y finales del s. XIX para que ingleses y franceses sobre todo, pero también más tarde alemanes, escandinavos y norteamericanos, se sientan atraídos por los monumentos y las huellas de la cultura islámica en las provincias del sur de España, prácticamente los únicos y mejor conservados en Europa, y que mostraban además un contenido cultural exótico dentro de un país no musulmán.

Mucho más tarde, en 1987 y ya sumergidos en el modelo turístico del sol y playa dominante en toda la Europa mediterránea, la UNESCO aprueba el Camino de Santiago dándole el honor de ser el primer Itinerario Cultural Europeo, más tarde declarado Patrimonio de la Humanidad. Con esa aprobación, la UNESCO asumía el compromiso de fomentar la investigación histórica sobre temas jacobeos, de mejorar fisicamente el Camino y de coordinar la restauración del patrimonio a lo largo de su trazado, pero al mismo tiempo iniciaba un proyecto a muy largo plazo para fomentar los intercambios y, en cierto modo, el multiculturalismo, entre todos los países, regiones y comunidades locales implicadas geográficamente en el Camino. Esto implicaba en rigor una política de desarrollo local sobre la base del patrimonio monumental y antropológico inmaterial, que podía implicar, como así sucedió en muchos lugares, cambios sociales, económicos y nuevos liderazgos.

Ese espíritu de identificar y potenciar vías de participación social y de establecer pautas de solidaridad entre comunidades locales interrelacionadas en un proceso común de influencias recíprocas dio lugar a la creación en 1998 del Comité Científico Internacional de Itinerarios Culturales (CIIC) que, entre otros cometidos, tiene el de inventariar y catalogar recursos que puedan servir para elaborar y promocionar un itinerario cultural. Los resultados de esta acción institucional son irregulares, teniendo en cuenta que presentan una notable pluralidad de dimensiones que trasciende a su función primitiva y of recen la posibilidad de poner en marcha nuevas dinámicas de cooperación cultural y económica entre los pueblos, no siempre entendidas de forma coincidente, aunque permitan una lectura plural y realista de la historia y renueven las hipótesis científicas para acrecentar y actualizar el conocimiento y las propias identidades culturales de aquellos pueblos.
Por esas razones los itinerarios culturales ayudan a modificar la escala de evaluación y reconocimiento social del patrimonio cultural y sirven como instrumento para modif icar igualmente la identidad de las comunidades locales, revelando una dimensión que trasciende a su significado local y la transforma en expresiones de ámbito universal. Esto llega a traducirse en la extensión de la agilidad con que los actores sociales se movilizan desde una perspectiva solidaria para intercambiar proyectos de renovación que pueden afectar a todo el cuerpo social, y eso se muestra especialmente importante para todas las culturas implicadas en el trayecto de los itinerarios, ante la confusión y la pérdida de conciencia de la autenticidad histórica que acompaña en este momento al proceso de globalización y a la dominación de una falsa cultura urbana, movida casi en exclusiva por el beneficio empresarial.

Desde una visión coincidente pero con mayor contenido social, la Unión Europea mantiene un criterio de apoyo al turismo cultural considerando que, además de consolidar las propias identidades de los pueblos, constituye también una cantera de actividades económicas y de creación de nuevos puestos de trabajo, lo que explica que la principal fuente de financiamiento europeo a proyectos culturales sean los Fondos Estructurales. Esa política de protección está fundamentada en la evidencia de que los proyectos culturales sirven para generar dinámicas de desarrollo local, sobre todo a través de tres vías (COBO, 2001):

j Creando o manteniendo empleo en la industria de la cultura; por ejemplo, poniendo en marcha un proyecto de museo etnográfico o en ámbitos relacionados con la preservación, evaluación social y uso del patrimonio histórico. Varios casos ilustran esta acción, como la creación de of icios de restauradores de órganos barrocos, a partir del programa de restauración de los órganos de monasterios de Castilla-León con fondos europeos.

j Mejorando la posición de una región determinada y consiguiendo que incremente su atractivo para atraer inversiones empresariales, teniendo en cuenta que cada vez toma más importancia el nivel cultural de una comunidad en las decisiones de localización de nuevas inversiones. En este momento, la cultura constituye un factor de relevancia para la renovación de zonas industriales en declive o fuera de los circuitos económicos principales, y el caso del Museo de la Minería en Asturias puede ser un ejemplo importante para descubrir que en la sociedad postindustrial la cultura es un instrumento fundamental para la transferencia de conocimientos.

j Facilitando el acceso o la reincorporación al mercado de trabajo de personas que por su edad o por su formación tienen dificultades para emplearse, y también aquí el caso del Museo Minero de la comarca de Berguedá configura ot ro ejemplo de recolocación laboral de los trabajadores excedentes y sus familias. 


\section{LOS ITINERARIOS CULTURALES EN LA EURORREGIÓN}

Como en los casos citados anteriormente, casi la totalidad de los proyectos de turismo cultural en Galicia y Portugal están unidos a ayudas europeas, especialmente a los programas Leader I y II. Comentaremos aquí dos casos que integran recursos naturales y culturales bien diferenciados, y que desde nuestro enfoque podrían suponer a medio plazo una extensión axial de los itinerarios, uniendo las ofertas de las dos regiones y reconfigurando el territorio también desde la actividad turística.

La iniciativa Terra de Trives, en el sur de Ourense, contempla una elevada diversidad de actuaciones, intentando aprovechar el mayor número de recursos del territorio. El proyecto nació a partir da declaración de Trives como comarca piloto para poner en marcha desde 1994 un Plan de Desarrollo Comarcal de la Xunta de Galicia (del que curiosamente nunca se presentaron cuentas al Parlamento), y que contó con un relevante apoyo de recursos financieros y humanos de la administración, después de crearse en 1994 la Fundación Comarcal y más tarde el Patronato de Turismo Terra de Trives. Estas entidades realizaron un estudio previo de la comarca, elaborando unas líneas generales de actuación estratégica en las que el turismo se convertía en la actividad central que dinamizaba a todos los demás sectores de la economía local (ÁLVA REZ et al., 2002).

Con todo, y a pesar de marcar como objetivo la creación de redes en su programación inicial de actuaciones y actividades, y de estructurarse como un itinerario cultural-natural, los proyectos concretos llevados a cabo presentan un aspecto más bien irregular y no claramente vertebrador del territorio. Puede verse así que se pusieron en marcha acciones como:

j El Centro Comarcal Expo-Trives, exposición permanente e informativa de los recursos de la comarca.

j El Aula de la Naturaleza, ligada a una ruta de senderismo de uso libre.

j Un Centro de Interpretación Histórico-Cultural, pensado más como museo que como centro de apoyo al turismo activo en la comarca.

j El Centro de la Identidad Gallega, con una operatividad semejante a lo anterior y de reducidas dimensiones y contenido, a pesar de su espectacular denominación.

j La recuperación de la calzada romana que venía desde Astorga, pensada como una ruta temática ligada al patrimonio que dejó Roma en estas tierras.

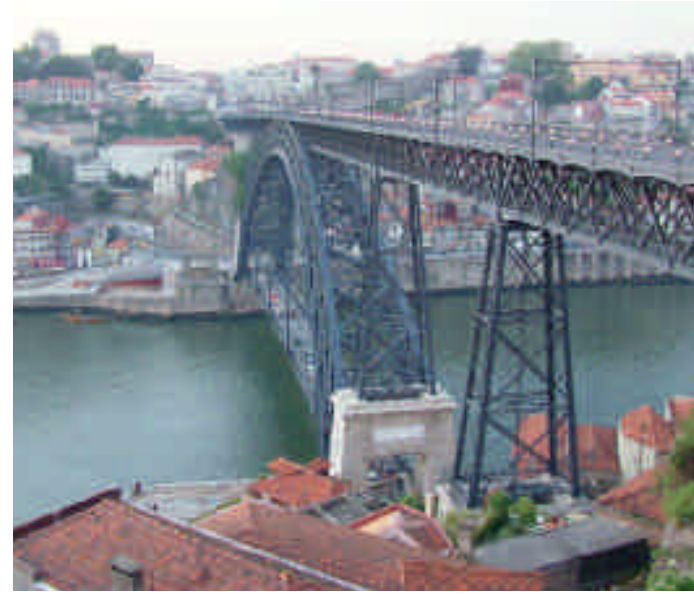

W Puente Don Luís I, construido en 1886 por discípulos de Gustave Eiffel; sus dos tramos comunican los barrios alto y bajo de la ciudad de Oporto / Isabel Dugo 


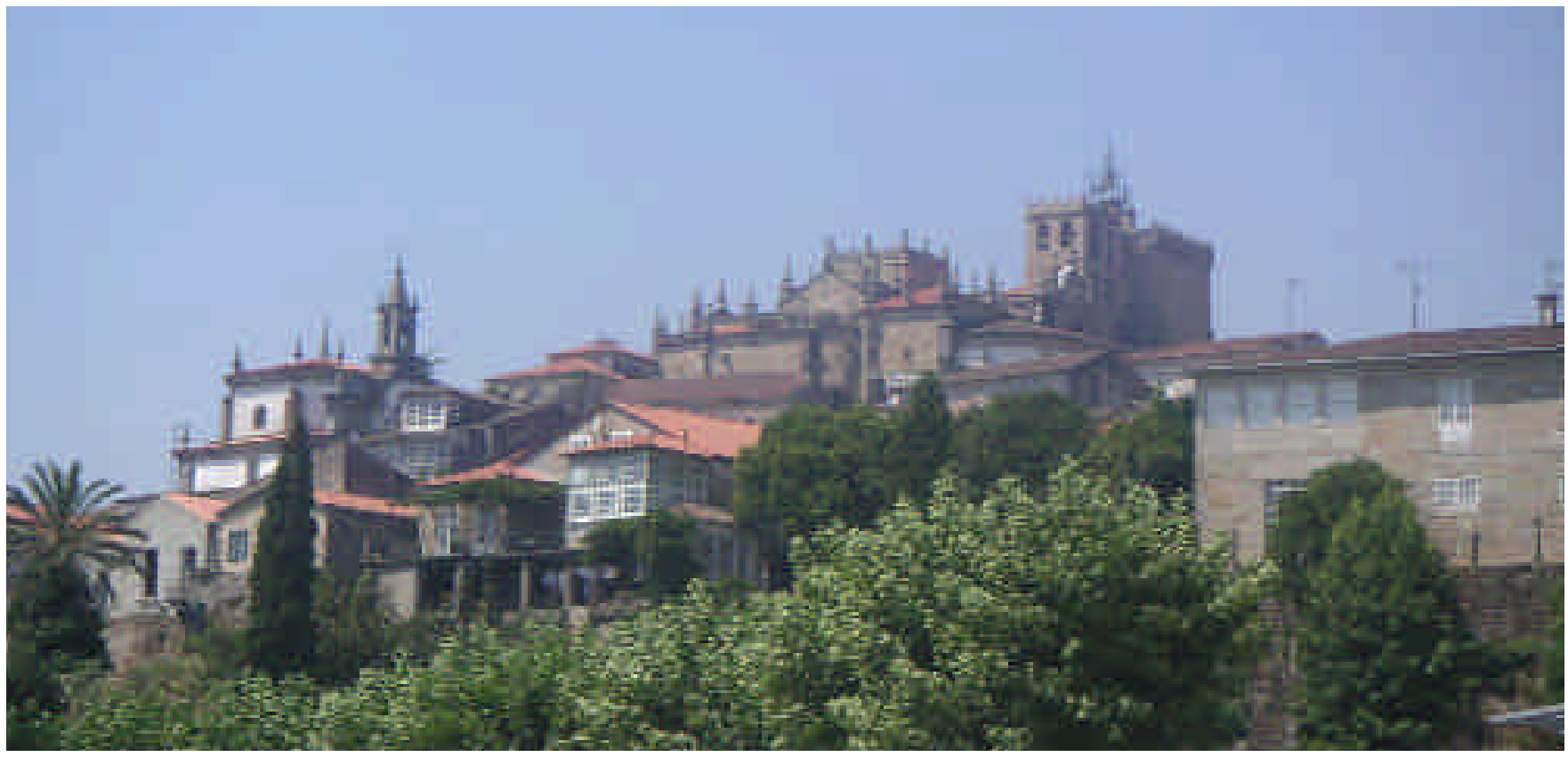

W Catedral-fortaleza de Tui (Pontevedra, Galicia), ciudad fronteriza con Portugal, vista desde el río Minho. La Fortaleza de Valença do Minho es el correlato de la de Tui al otro lado del río, ya en tierras portuguesas / Beatriz Castellano

\section{Los itinerarios culturales sirven como instrumento para modificar la identidad de las comunidades, revelando su dimensión universal}

j El proyecto Teatro en los Caminos, que busca la colaboración de jóvenes grupos dramáticos gallegos y que se extendería a todos los municipios de la comarca, con obras tradicionales, populares y propias.

La singularidad de este proyecto fue la interrelación de tres tipos de actividades raramente coincidentes en Galicia: las económicas tradicio nales (agricultura, especialmente viñedo y artesanía), el turismo deportivo de invierno de una empresa claramente consolidada (la estación de esquí-montaña de Manzaneda) y las nuevas actividades y propuestas llevadas a cabo dentro del plan comarcal, lo que en principio debería dar como resultado el establecimiento y la consolidación de nuevas redes de atracción y una mayor vertebración del territorio.

Estos objetivos fueron irregularmente conseguidos por varias razones, entre las que se podrían señalar, por una parte, la falta de un objetivo vertebrador claro en las actuaciones propuestas (por ejemplo, redes de relación entre las actividades dentro del territorio) y, por otra parte, la escasa interrelación entre la identidad cultural de la comarca (que no siempre aparece explícita en las rehabilitaciones de largo recorrido histórico, como sería el caso de la ruta romana) y los agentes protagonistas de los proyectos más promocionados (el alcalde y los funcionarios del plan comarcal). 
Sin embargo, el mayor interés de este proyecto es su proyección hacia el sur, que permitiría extender la of erta cultural hacia la vecina comarca de Chaves, en Portugal, igualmente involucrada en un programa Leader de desarrollo local, sobre la base de sus recursos termales e históricos, continuando el itinerario por Pedras Salgadas y Vila Real hasta el río Douro, donde enlazaría con la actual of erta económico-etnográfica de la ruta del traslado del vino de Trás-Os. Montes hacia Porto.

El otro proyecto, en el que participó el autor como director del estudio previo, fue una iniciativa de la Dirección General de Turismo de la Xunta para crear un Plan Integral de Turismo en la Comarca del Baixo Miño, área que presentaba importantes recursos y una situación climática y antropológica singular, en la desembocadura del río Miño y en el paso del Camino de Santiago portugués, además vecina del destino turístico ya consolidado de Rías Baixas.

La comarca está integrada por cinco municipios (Tui, Tomiño, o Rosal, A Guarda e Oia) y, evidentemente, el elemento más característico de su territorio, que marcó históricamente sus relaciones sociales y también su cultura, es el río Miño, frontera natural con el vecino país de Portugal, que en la comarca del Vale do Minho tiene la réplica de su identidad cultural. Como todo río atlántico, los efectos de las mareas llegan hasta 38 km río arriba, permitiendo la navegación y el aprovechamiento pesquero de su fauna, y causando en tiempos pasados serios conflictos fronterizos por esa causa.

Siempre en relación con el río, sus recursos naturales e históricos aparecen ligados y entrelazados unos con otros sin tener en cuenta en ningún caso la artificialidad de la frontera administrativa, que también es ignorada en este momento por los turistas que visitan la comarca (PARDELLAS y PADín, 2001). Entre los relacionados con la historia y la cultura más relevantes pueden destacarse:

j El Parque de Santa Tegra, en A Guarda, declarado en 1931 Monumento Nacional Histórico-Artístico, y considerado como uno de los yacimientos arqueológicos de la época castrexa más importantes de España.

j El propio Camino de Santiago portugués que, por la frecuencia de los conflictos bélicos entre portugueses y españoles a lo largo de los siglos XVII y XVIII, tenía varios puntos de cruce del río, en Valença do MinhoTui, Vilanova de Cerveira-Goián o Caminha-A Guarda, en función de la menor peligrosidad para los peregrinos.

j La unión de las ciudades Tui-Valença do Minho, pendiente de aprobación como conjunto histórico de interés cultural europeo.

j Los molinos de agua, que tienen una presencia generalizada en toda la comarca.
Y entre los relacionados con la naturaleza deben señalarse:

j El Parque del Monte Aloia, declarado Sitio Natural de Interés Nacional en el año 1935, el primero declarado en Galicia y acogido en la actualidad a la figura de protección de Parque Natural.

j La Sierra do Argalo, entre los Ayuntamientos de 0 Rosal, Oia y Tomiño, de propiedad comunal, con una masa forestal de especial interés.

j Las Gándaras de Budiño, en la orilla del río Louro, ecosistema de gran valor ecológico por su vegetación y fauna, y que como atractivo agregado constituye la última zona húmeda de agua dulce que queda en Galicia, después de la desecación de la laguna de Antela en Ourense en los años 60 .

j El Estuario del Miño y, por extensión, las dos orillas del Miño, declaradas por la UE como Lugar de Interés Comunitario (LIC), y que por su vegetación autóctona constituye un ecosistema singular en el conjunto de la Eurorregión.

Sin duda, el recurso turístico común básico era justamente el río, que pasaba a constituir en el proyecto el nodo fundamental de la comarca, con su doble valor natural e histórico, esto es, como patrimonio paisajístico con todos sus elementos vivos e inertes (riqueza faunística, humedales y playas) y, en paralelo, como elemento de unión de todo el patrimonio histórico-cultural de las dos comunidades vecinas, con sus vestigios arqueológicos, construcciones militares derivadas de los conflictos bélicos entre Portugal y España desde el s. XVII y, finalmente, como área fronteriza, lo que agregaba valor turístico a todo el proyecto.

A lo largo del río aparecen tres singulares complejos de atracción fronterizos, presentando una estructura de oferta común en ambos márgenes, con vías de comunicación igualmente comunes (la mayoría construidas con ayudas europeas del programa INTERREG) y con servicios de características muy homogéneas. Son espacios integrados por ciudades enfrentadas geográficamente por el río, mostrando un patrimonio monumental histórico muy semejante, como resultado de los citados conflictos bélicos, pero también por sus relaciones económicas en tiempos de paz, y aparecen así definidos por unas características sociales y culturales complementarias entre sí. Destaca la configuración del citado conjunto monumental de Tui-Valença y las fortalezas y playas de Goián-Vila Nova y A Guarda-Caminha, que constituyen al mismo tiempo los tres puntos de cruce del río actual, a través de puentes, en los dos primeros casos, y por un ferry, en el caso de A GuardaCaminha, pero que fueron a su vez los pasos de cruce de los itinerarios del Camino Portugués. 
Desde esa perspectiva, la propuesta global apuntaba a la creación de una ruta temática circulatoria principal a lo largo del río, que integraría aspectos históricos (las vivencias comunes de los dos pueblos), y donde ocuparía un lugar preferente el Camino de Santiago Portugués, pero también las identidades culturales del territorio conjunto (que en realidad es una sola identidad con formas diferenciadas en gallego y portugués). De forma paralela se proponían dos rutas temáticas de naturaleza e historia en el Baixo Miño gallego, uniendo el Parque Natural del Monte Aloia con la Sierra do Argalo, y la segunda uniendo también el Parque de Sta. Tegra con el Estuario del Miño, que contemplaban de forma integrada los recursos naturales más singulares e identificables de la comarca con su historia, dado que podían visitarse las reconstrucciones de los poblados castrexos más antiguos del norte de la Península.

El proyecto no llegó a llevarse a cabo en su totalidad de forma estructurada, entre otras razones por falta de liderazgo local y también por falta de interés de las instituciones autonómicas en su extensión al ámbito de la Eurorregión. Eso no impidió que se fueran acometiendo varias actuaciones aisladas por iniciativa de los alcaldes, más o menos interrelacionadas con sus vecinos portugueses (por ejemplo, en el caso de Tui-Valença), y el resultado es por ahora un conjunto de of ertas turísticas locales de notable atractivo, pero escasamente interrelacionadas y de ningún modo estructuradas en red como un itinerario orientado a recuperar y dar valor social a la identidad y la cultura común de los dos pueblos.

En los dos casos destacamos varios elementos comunes que implican al potencial turístico de la Eurorregión y a su papel en el desarrollo local de áreas de interior:

j Usan conjuntamente el patrimonio natural y cultural, pero así como en el proyecto del Baixo Miño hay una referencia clara de itinerario cultural, desde la consideración del territorio común y del río Miño como factor integrador de las identidades de los pueblos gallego y portugués en el de Terra de Trives todavía persiste la visión local y se ignoran otras posibilidades futuras de aprovechamiento conjunto de los recursos, sin aparecer una apuesta semejante de itinerario.

j La recuperación de la identidad de la cultura local es un factor básico en la exposición teórica de los dos proyectos, pero apenas hay luego una atención específica a los procesos de cambio necesarios en las comunidades rurales, y en concreto en el afianzamiento de los liderazgos, para que el legado cultural de la memoria actúe como motor del desarrollo local en apoyo de las actuaciones económicas.

j El Camino de Santiago es en rigor un recurso y atracción fundamental para el segmento de demanda interesado en su papel como factor de mestizaje multicultural, o bien simplemente en su efecto religioso. A parece citado en los dos proyectos, pero en ningún caso tuvo una atención especial en las actuaciones concretas de la administración.

\section{A MODO DE ELEMENTALES CONCLUSIONES}

Del análisis anterior puede deducirse que es posible usar los recursos naturales, históricos y antropológicos de un territorio rural para crear y mantener empleo con actuaciones apropiadas para un uso sostenible, consiguiendo además una mayor valoración social del patrimonio cultural, siempre que la identidad de las comunidades sociales constituya el objetivo básico del conjunto de los proyectos.

Por otra parte, esas mismas actuaciones llegan a conseguir igualmente mejoras de diverso tipo en una comarca o municipio (infraestructuras, equipamiento), haciendo que incremente su atractivo para atraer inversiones empresariales, y teniendo en cuenta que cada vez toma más importancia el nivel cultural de una comunidad en las decisiones de localización de nuevas inversiones, lo que demuestra que, en este momento, la cultura constituye un factor de relevancia para la renovación de las zonas agrícolas o incluso industriales en declive, o fuera de los circuitos económicos principales.

Es posible también pensar en el uso de los recursos comunes en la Eurorregión Galicia-Norte de Portugal, tanto por el propio comportamiento actual de los turistas que visitan las dos regiones, como por el potencial de incremento de la atracción a medio plazo de un destino conjunto con dos ejes de itinerarios culturales.

El It inerario Jacobeo, Camino de Santiago, declarado primer Itinerario Cultural Europeo en 1987, y luego Patrimonio de la Humanidad por la UNESCO, debía servir en gran medida para aquellos objetivos, y de hecho, la propia Unión Europea apoya con fondos financieros importantes el compromiso de fomentar la investigación histórica sobre temas jacobeos, de mejorar físicamente el Camino y de coordinar la restauración del patrimonio a lo largo de su trazado. Pero en todo caso, corresponde a las autoridades de las regiones que atraviesa su trazado y, por lógica, a los líderes locales, la elaboración de proyectos para aprovechar adecuadamente este recurso en cada territorio, para fortalecer su identidad cultural y, al mismo tiempo, para fomentar los intercambios y, en cierto modo, el multiculturalismo entre todas las comunidades locales implicadas geográficamente en el Camino. 


\section{Bibliografía}

AECIT (2005) La actividad turística española en 2004. Madrid: AECIT, 2005

ÁLVAREZ CAO, R. et al. (2003) Terra de Trives, experiencia de turismo en una comarca de montaña. EnActas VIII Coloquio: la geografía y la gestión del turismo. Santiago: Universidad, Servicio de Pubicaciones, 2003, pp. 105-113

ASHWORTH, G. J., DIETVORST, G. J. (ed.) (1995)Tourism and Spatial Transformations. Implications for Policy and Planning London: CAB International, 1995

AYDALOT, P. (1986) Milieux innovateurs en Europe. Paris: GREMI, 1986

BASELGA, J. (2003) Las estrategias de mercado en la promoción turística de las ciudades monumentales. En Actas Congreso Desarrollo Turístico Integral de Ciudades Monumentales. Granada: Patronato Provincial de Turismo, 2003

BAYON, F. (dir.) (1999) 50 años del turismo español: un análi sis histórico y estructural. Madrid: Centro de Estudios Ramón Areces, D.L., 1999

BORRELL, D. (2001) La Ruta Jacobea: intercambio y cultura. En Actas Congreso Internacional de Itininerarios Culturales. Madrid: Ministerio de Cultura, 2001

BRITTON, S. G. (1980) A conceptual model of tourism in a peripheral economy. En PEARCE, D. (ed.) Tourism in the South Pacific: the contribution of research to development and planning. N.Z. MAB Report no 6, National Commission for UNESCO/Department of Geogrphy, University of Canterbury, Chrtstchurch, pp. 1-12

CADIMA, J. et al. (2002) 0 turismo no espaço rural: uma digressâo pelo tema a pretexto da situaçâo e evoluçâo do fenómeno en Portugal. En Actas do VIII Encontro Nacional da APDR. Coimbra: Colecçâo APDR, 2002

CAMPESINO FERNÁNDEZ, A.; VELASCO BERNARDO, C (coord.) (1996) Portugal-España: ordenación territorial de suroeste comunitario. Acta, ponencias y comunicaciones del VII Coloquio Ibérico de Geografía. Cáceres: Servicio de Publicaciones de la Universidad de Extremadura, 1996

COBO, R. (2001) Un recurso para el desarrollo de las regiones. En Actas Congreso Internacional de Itinerarios Culturales. Madrid: Ministerio de Cultura, 2001

COFFEY, W.J.; PÒLESE, M. (1985) Local development: conceptual basis and policy implications. Regional Studies, $n^{\circ} 19$ 1985, pp. 85-93

DE MIGUEL, A. (2000) Imagen social recíproca de españoles y portugueses de la Raya.Zamora: Fundaçâo Afonso Henriques, 2000

DREDGE, D. (1999) Planificación y diseño de destinos turísti cos. Annals of Tourism Research en español, v.1, no 2, 1999, pp. 394-414

FAGANCE, M. (1995) Changing Paradigms of Orthodoxy: The Case of Spatial Models in Tourism Planning. Les Cahiers du Tourisme. Aix-en-Provence: Centre Des Hautes Etudes Touristiques, 1995
FUA, G. (1988) Small-scale industry in rural areas: the Italian experience. En ARROW, K. J. (ed.) The Balance between Industry and Agriculture in Economic Development. London: Macmillan, 1988

FYALL, A.; GARROD, B. (1998) Sustanaibility and Rura Heritage. En May, D. and O'Halon, L. (ed.) Rural Tourism Management: Sustainable Options, Conference Proceedings (9-12 septiembre 1998). Scottish Agricultural College, Auchenvyre

GAROFOLI, G. (1992) Endogenous development and southern Europe. Aldeshot: Avebury, 1992

GETZ, D. (1986) Models in Tourism planning. Tow ards integration of theory and practice. Tourist management, v. 7, no 1, pp 21-32

GUNN, G. A. (1972) Vacationscape: Designing Tourist Regionals. Austin: University of Texas, 1972

GUNN, G. A. (1993) Tourism Planning: basics, concepts, cases. London: Taylor\& Francis, 1993

HERRERO DE LA FUENTE, A. (2002) La cooperación transfronteriza Hispano-Portuguesa en 2001. Madrid: Tecnos, 2002

INSKEEP, E. (1988) Tourism Planning: An Emerging Epcecialization. Journal of the American Planning Association no 54, pp. $360-372$

INSKEEP, E. (1991) Tourism planning. An integrated and sustainable approach. New York: Van Nostrand Reinhold, 199

LEIPER, N. (1995) Tourism Management. Melbourne: RMIT Press, 1995

LUE, C.; CROMPTON, J. L.; FESENMAIER, D. R. (1993) Conceptualization of Multidestination Pleasure Trips. Annals of Tourism Research, 20, 1993, pp. 289-301

LUNDGREN, J. (1982) The Tourism Frontier of Noveau Québec: functions and Regional Linkages. Tourism Review, $\mathrm{n}$ 37,1982, pp. $17-35$

MAILLAT, D. (1998) Interaction between urban systems and localized productive system. European Planning Studies, $n 06$, 1998, pp.117-129

MANNING, T. (1999) Indicators of tourism sustainability Tourism Management, v. 20, 1999, pp.179-181

MARTÍNEZ PALLARÉS, P. (2000) El gobierno local como ámbito de gestión turística. En Actas III Congreso universidad y empresa. Valencia: Tirant lo Blanch, 2000, pp. 69-87

OPPERMAN, M. (1993) Tourism Space in Developing Countries. Annals of Tourism Research, no 20, 1993, pp. 535 560

PARDELLAS DE BLAS, X. (2002) Aproximación á actividade turística nas cidades do Eixo Atlántico. Vigo: Ed. Eixo Atlántico, 2002

PARDELLAS DE BLAS, X. et al. (2003) El desarrollo local y la cooperación ente España y Portugal. En Actas do IX Encontro Nacional da APDR. Coimbra: Colecçâo APDR, 2003
PARDELLAS DE BLAS, X.; PADÍN, C. (2001) El patrimonio etnográfico y el desarrollo local: El Baixo Miño como destino turístico en Galicia. IV Congreso De Turismo, Universidad Y Empresa. Castellón

PEARCE, D. (1995) Tourism today. A geographical analysis (2nd ed.). New York: Longman, 1995

PIZAM, A.; MANSFELD, Y. (ed.) (2000) Consumer behavior in travel and tourism. Binghamton: Haw orth Press

RITCHIE, J. A.; CROUCH, G. (2003) The competitive destination: A sustainability perspective. Canada: University of Calgary, 2003

ROBERTS, L.; HALL, D. (ed.) (2001) Rural tourism and recreation: principles to practice. Oxon and New York: $C A B$ Publishing, 2001

RUIZ, J. A. (1999) Estudio de mercados turísticos emisores a Portugal. Madrid: Turespaña, 1999

RYAN, C. (1999) Issues of sustainability in tourism. Tourism Management, no20, pp. 177-192

SOUTO, X. M. et al. (2005) Segundo estudio estratéxico do Eixo Atlántico. Vigo-Porto: Ed. Eixo Atlántico

SWARBROOKE, J.; HORNER, S. (1999) Consumer Behavior in Tourism. Oxford: Butterw orth Heinemann, 1999

VALDÉS, L.; RUIZ, A. (coord.) (1996) Turismo y promoción de destinos turísticos: implicaciones empresariales. Oviedo: Universidad, Servicio de Publicaciones

VÁZQUEZ BARQUERO, A. (2005) De las nuevas fuerzas del desarrollo. Barcelona: Bosch, 2005

VÁZOUEZ BAROUERO, A. (1999) Desarrollo, redes e innovación. Lecciones sobre desarrollo endógeno. Madrid: Pirámide, 1999

VENADE, N. (2004) As euro-regiôes e o futuro da Europa: 0 modelo da euro-regiâo Galiza-Norte de Portugal. Porto: Ed. Eixo Atlántico,2004

VERA, F. (coord.) (1997) Análisis territorial del turismo. Barcelona: Ariel

VERA,F. (2002) Estrategias de diversificación y diferenciación en destinos turísticos litorales. En Pardellas, X. (dir.) Estrategias turísticas urbanas. Vigo: Universidad 